\title{
Young adult non-smokers' exposure to real-world tobacco marketing: results of an ecological momentary assessment pilot study
}

Shyanika W. Rose ${ }^{1 *}$, Andrew Anesetti-Rothermel ${ }^{1}$, Hoda Elmasry ${ }^{1}$ and Ray Niaura ${ }^{1,2}$

\begin{abstract}
Background: The aims of this pilot study were to assess and characterize non-current smoking young adults' exposure to tobacco marketing through an ecological momentary assessment protocol.

Methods: Ecological momentary assessment (EMA) consists of repeated measurement of momentary phenomena and is well-suited to capture sporadic experiences in the real-world, such as exposure to tobacco marketing. EMA has the potential to capture detailed information about real-world marketing exposures in ways that reduce recall bias and increase ecological validity. In this study, young adults $(n=31$; ages $18-25)$ responded to random prompts regarding their momentary exposure to tobacco marketing via text messages on their smartphones for 14 days ( $n=1798$ observations). Unadjusted and adjusted analyses were conducted using multilevel logistic regression to assess the odds of exposure accounting for correlation of multiple repeated measures within individuals while controlling for variability between individuals.
\end{abstract}

Results: Respondents reported, on average, two momentary exposures to tobacco advertising in the 14-day study period. In adjusted analyses, African-American (aOR 3.36; $95 \% \mathrm{Cl} 1.07,10.54)$ and Hispanic respondents (aOR 5.08; $95 \% \mathrm{Cl} 1.28,20.13$ ) were more likely to report exposure to tobacco advertising. Respondents were also more likely to report exposure when also exposed to others using tobacco products and when they were at stores compared with at home (aOR 14.82; 95\% Cl 3.61,60.88).

Conclusion: Non-smoking young adults report exposure to tobacco marketing particularly at the point-of-sale, with the highest likelihood of exposure among African-American and Hispanic young people. EMA protocols can be effective in assessing the potential impact of point-of-sale tobacco marketing on young adults.

Keywords: District of Columbia, Marketing, Pilot projects, Tobacco, Young adult, Health equity

\section{Background}

Exposure to tobacco marketing has been associated with increased risk of smoking in youth $[1,2]$ and young adults $[3,4]$. Individual exposures throughout time and places are cumulative and can change positive expectancies and normative beliefs about tobacco products [5]. Exposure to tobacco marketing in real world settings affects many facets of tobacco use, but its role in tobacco use initiation among non-users is not well understood [6, 7]. Exposure

*Correspondence: srose@truthinitiative.org

1 Schroeder Institute at Truth Initiative, Washington, DC, USA

Full list of author information is available at the end of the article to tobacco marketing may also be more prevalent among minority populations. For instance, there are significantly more menthol advertisements in areas with higher proportions of African-American residents [8,9] and near schools with more African American students [10]. Exposure to tobacco marketing and brand recognition is higher among non-smoking African-American [11, 12] and Hispanic [12] youth and young adults and tends to be associated with greater risk of smoking initiation. Identifying how young adult non-smokers are exposed to tobacco marketing can help to understand who is at risk of exposure and potentially future tobacco use. 
This study utilized a real-time and ecologically valid measure of exposure to tobacco advertising through ecological momentary assessment (EMA) [13, 14]. EMA can provide real-time tracking of exposure to and attitudes towards tobacco advertising closer to the moment of exposure, rather than through retrospective recall [1, 15, 16]. Prior EMA studies have examined self-reported overall tobacco advertising exposure $[3,17]$ or exposure to POS tobacco outlets [18] However, few studies have focused on exposure of non-current smokers to tobacco marketing. One study of college students found that almost $70 \%$ of self-reported exposure to tobacco marketing measured through an EMA protocol was at point of sale [19], and that exposure was related to future risk of smoking among both smokers and non-smokers [3]. The current study tested a protocol to assess real time exposure to tobacco advertising through EMA and identified correlates of exposure.

\section{Methods}

\section{Ethical considerations}

The study was approved by the Chesapeake institutional review board (Study Number \#Pro00012367). Respondents were provided with informed consent to participate and identifiable information was kept separate from respondent survey and EMA text message responses. The EMA data was transmitted via standard text messages (SMS) via a respondents' smartphone to a secure database. In the EMA, respondents were simply asked to provide numeric responses to questions without providing other identifiable information. In transit, SMS messages are encrypted. Once received, the data were stored in a secure firewall-protected and password-protected database via an encrypted representational state transfer (REST) application programing interface with access only to authorized users.

\section{Recruitment}

As part of an EMA pilot study for young adult nonsmokers that also examined store visiting behavior (not reported in this study), participants were recruited in summer 2015 via online and print advertisements (e.g., Craigslist, local newspaper, and flyers). In total, 258 individuals responded to the screener within a 1-month period. Eligible respondents were (1) between the ages of 18-25, (2) did not smoke cigarettes in the past 30 days, (3) lived and worked/attended school in Washington, DC, (4) owned their own android or iPhone smartphone with an unlimited text message plan, and (5) agreed to participate in the study $(\mathrm{n}=31)$. This pilot protocol was developed to determine the feasibility of engaging young adult non-smokers in reporting on their exposure to tobacco marketing and store visiting building, in part, on an approach used in a prior study of smoker's relapse and store visiting used by members of this study team [18].

\section{Data collection}

All 31 participants completed a baseline survey of participant demographics, tobacco use history, and susceptibility to future cigarette use (see Additional file 1). Respondents downloaded the study application to their phone which enrolled them in a text message protocol. Over the next 14 days they were randomly prompted 6 times a day about their exposure to tobacco advertising. As a result, each respondent was assessed over 80 times. They had $1 \mathrm{~h}$ to respond to each prompt via text message before it expired. All 31 respondents completed the EMA period.

\section{Measures}

Participant baseline measures included gender, race/ethnicity (White, African-American, Hispanic, other), past 30-day cigarette smoking status $(\mathrm{y} / \mathrm{n})$, age, self-reported personal financial status (live comfortably, meet needs with a little left, just meet basic needs, don't meet basic needs), and smoking status as ever (even one puff) or never. They were also asked about their susceptibility to future cigarette use using the validated three-item susceptibility to smoke index measured on a four point scale of definitely yes, probably yes, probably no, and definitely no ("Do you think in the future you might experiment with cigarettes?," "At any time in the next year do you think you will smoke a cigarette," and "If one of your best friends were to offer you a cigarette, would you smoke it?") [20]. Respondents who answered definitely no to all three questions were deemed non-susceptible, all others were deemed susceptible. EMA prompts measured tobacco marketing exposure as the outcome ["In your current location, do you see any advertisements or signs promoting tobacco?" $(\mathrm{y} / \mathrm{n})]$, current location (home, work, other's home, bar/restaurant, vehicle/in transit, outside, store, other), and exposure to tobacco use ("Is anyone using tobacco products in your current location?" Yes, in view, yes, in my group, no) adapted from a prior study [17]. Participants self-defined what constituted tobacco marketing.

\section{Analysis}

Descriptive statistics were used to characterize participants in terms of sociodemographic factors, smoking status and EMA responses. The analysis utilized multilevel logistic regression modeling using Stata version 13 in a two-level model, to account for clustering of multiple EMA responses within individual respondents. We anticipate that responses by the same individual are correlated and thus a multilevel modelling approach 
properly adjusts standard errors and associated confidence intervals. Additionally, multilevel models using repeated measure data allows for including all subjects' observations despite the potential for missing data points for incomplete response to the EMA by participants. We modeled the likelihood of respondent momentary exposure to tobacco advertising when responding to the random prompts. Multilevel modeling extends to binary outcomes by using a logit link function, in which the log odds of momentary exposure to tobacco advertising was modeled as a linear combination of correlates. We first estimated the null model (a model with no predictors) finding that the logistic multilevel model was preferable to an ordinary logistic regression model based on a likelihood ratio test provided by STATA showing a highly significant result $\left[x^{2}(1) 28.99 p=0.00\right]$. We report $95 \%$ confidence intervals with significance levels set at the $\mathrm{p}<0.05$ level for all statistical analyses. Correlates of exposure included gender, race/ethnicity, financial situation, baseline cigarette susceptibility, location when responding to EMA prompts, and momentary exposure to smoking. We report both bivariate crude odds ratios and adjusted odds ratios in the multilevel logistic regression models along with $95 \%$ confidence intervals reporting significance at the $\mathrm{p}<0.05$ level. We retained all correlates in the adjusted model regardless of significance in the bivariate analyses to examine the potential joint effects of these variables on advertising exposure.

\section{Results}

The respondents (Table 1 ) were predominantly female (74\%) and African-American (58\%). Respondents were highly educated with $61 \%$ having a Bachelor's degree or higher and had a comfortable personal financial situation with $65 \%$ meeting needs with a little left or living comfortably. The majority (66\%) had ever had even a puff of a cigarette but only one respondent had smoked 100 lifetime cigarettes. The remaining participants were never cigarette smokers. At baseline, $48 \%$ were susceptible to future cigarette use.

During the EMA, the respondents were asked a total of 2560 random prompts, responding to $73 \%$ and completing $70 \%$. The average number of complete random prompts was 58 per subject (median 59, range 25-75). On average, over the 14 day EMA period, respondents reported 1.9 exposures to tobacco advertising (range $0-10)$.

For the null multilevel model of exposure to tobacco advertising, the intraclass correlation was 0.24 indicating that $24 \%$ of the variance in tobacco advertising exposure was accounted for by clustering of observations within participants. In unadjusted analyses (Table 2), Hispanic respondents, participants not meeting basic financial
Table 1 Descriptive statistics of participant characteristics and ecological momentary assessments

\begin{tabular}{|c|c|c|}
\hline & Mean/N & $\mathrm{SD} / \%$ \\
\hline \multicolumn{3}{|l|}{ Sociodemographics } \\
\hline Mean age & 22.5 & 1.9 \\
\hline Female & 23 & 74.0 \\
\hline \multicolumn{3}{|l|}{ Race/ethnicity } \\
\hline Non-Hispanic African American & 18 & 58.1 \\
\hline Non-Hispanic White & 9 & 29.0 \\
\hline Non-Hispanic Asian & 2 & 6.5 \\
\hline Hispanic & 2 & 6.5 \\
\hline \multicolumn{3}{|l|}{ Education } \\
\hline Some college, no degree & 11 & 35.5 \\
\hline Associate's degree & 1 & 3.2 \\
\hline Bachelor's degree & 18 & 58.1 \\
\hline Master's degree & 1 & 3.2 \\
\hline \multicolumn{3}{|l|}{ Financial situation } \\
\hline Live comfortably & 5 & 16.1 \\
\hline Meet needs with a little left & 15 & 48.4 \\
\hline Just meet basic expenses & 10 & 32.3 \\
\hline Don't meet basic expenses & 1 & 3.2 \\
\hline \multicolumn{3}{|l|}{ Smoking status ${ }^{a}$} \\
\hline Never & 11 & 35.5 \\
\hline Ever & 20 & 65.5 \\
\hline \multicolumn{3}{|l|}{ EMA responses } \\
\hline Completed & 1798 & 70.2 \\
\hline Abandoned & 83 & 3.2 \\
\hline Expired & 674 & 26.3 \\
\hline
\end{tabular}

a Smoking status defined as having ever tried at least one puff of a cigarette

needs, those exposed to tobacco use in their group or in their view, and respondents who were outside, at work or in a store had higher odds of being exposed to tobacco advertising.

Though based on small sample sizes, when adding covariates to the model (Table 2), African-Americans (aOR 3.36, 95\% CI 1.07, 10.54) and Hispanics (aOR 5.08, $95 \%$ CI $1.28,20.13$ ) had higher odds of reporting momentary exposure to tobacco advertising than Whites. Asians were no more likely to report exposure than Whites. Respondents who did not meet financial needs compared with those who lived comfortably were more likely to report exposure to advertising (aOR 6.12, 95\% CI 1.16, 32.37). Females also reported greater odds of exposure in adjusted analyses (aOR 3.92, 95\% CI 1.10, 13.96). Respondents were more likely to report exposure to tobacco advertising if someone in their group was using tobacco (aOR $17.04,95 \%$ CI 7.47, 38.87) or someone in their view was using tobacco (aOR 14.43, 95\% CI 6.38, 32.65). There were no significant differences in odds of reporting exposure based on baseline susceptibility to future cigarette 
Table 2 Unadjusted and adjusted multilevel logistic regression models of reported momentary exposure to tobacco advertising $\left(\mathrm{N}=1794^{\mathrm{a}}\right)$

\begin{tabular}{|c|c|c|c|c|c|c|c|}
\hline \multirow{3}{*}{$\begin{array}{l}\text { Variable } \\
\text { Female }\end{array}$} & \multirow{3}{*}{$\begin{array}{l}\%^{\mathbf{b}} \\
72.58\end{array}$} & \multicolumn{3}{|c|}{ Unadjusted model } & \multicolumn{3}{|c|}{ Adjusted model } \\
\hline & & \multirow{2}{*}{$\begin{array}{l}\text { OR } \\
1.67\end{array}$} & \multicolumn{2}{|c|}{$95 \% \mathrm{Cl}$} & \multirow{2}{*}{$\begin{array}{l}\text { aOR } \\
3.92\end{array}$} & \multicolumn{2}{|c|}{$95 \% \mathrm{Cl}$} \\
\hline & & & 0.51 & 5.42 & & 1.10 & 13.96 \\
\hline \multicolumn{8}{|l|}{ Race/ethnicity } \\
\hline Hispanic & 6.79 & 8.01 & 1.45 & 44.21 & 5.08 & 1.28 & 20.13 \\
\hline $\mathrm{NH}$ AA & 58.51 & 2.94 & 0.94 & 9.17 & 3.36 & 1.07 & 10.54 \\
\hline $\mathrm{NH}$ Asian & 6.90 & 0.63 & 0.05 & 8.13 & 1.60 & 0.14 & 18.29 \\
\hline White (ref) & 27.81 & - & - & - & - & - & - \\
\hline \multicolumn{8}{|l|}{ Personal financial situation } \\
\hline Live comfortably (ref) & 16.52 & - & - & - & - & - & - \\
\hline Meet needs with a little left & 46.55 & 0.95 & 0.27 & 3.35 & 0.47 & 0.15 & 1.46 \\
\hline Just meet basic needs & 33.65 & 1.43 & 0.39 & 5.23 & 1.06 & 0.34 & 3.35 \\
\hline Don't meet basic needs & 3.28 & 10.41 & 1.31 & 82.63 & 6.12 & 1.16 & 32.37 \\
\hline \multicolumn{8}{|c|}{ Baseline susceptibility to future tobacco use } \\
\hline Yes & 47.89 & 1.32 & 0.51 & 3.45 & 1.73 & 0.74 & 4.06 \\
\hline No (ref) & 52.11 & - & - & - & - & - & - \\
\hline \multicolumn{8}{|l|}{ Using tobacco } \\
\hline Yes, in my group & 2.89 & 17.05 & 7.60 & 38.24 & 17.04 & 7.47 & 38.87 \\
\hline Yes, in my view & 4.62 & 13.42 & 6.56 & 27.45 & 14.43 & 6.38 & 32.65 \\
\hline No (ref) & 92.27 & - & - & - & - & - & - \\
\hline \multicolumn{8}{|l|}{ Location } \\
\hline Bar/restaurant & 4.89 & 1.29 & 0.29 & 5.88 & 0.99 & 0.20 & 4.95 \\
\hline Other & 4.45 & 1.22 & 0.27 & 5.56 & 0.80 & 0.16 & 4.01 \\
\hline Others home & 7.17 & 1.58 & 0.41 & 6.07 & 0.95 & 0.23 & 4.01 \\
\hline Outside & 4.73 & 4.14 & 1.50 & 11.41 & 1.46 & 0.45 & 4.77 \\
\hline Store & 1.00 & 10.66 & 2.50 & 45.53 & 14.82 & 3.61 & 60.88 \\
\hline Vehicle/transit & 7.17 & 2.18 & 0.88 & 5.42 & 1.17 & 0.42 & 3.21 \\
\hline Work & 14.52 & 2.46 & 1.06 & 5.67 & 2.39 & 0.96 & 5.94 \\
\hline Home (ref) & 56.06 & - & - & - & - & - & - \\
\hline
\end{tabular}

Italic responses have $95 \%$ confidence intervals that do not include 1 and are significant at the $\mathrm{p}<0.05$ level of significance

${ }^{a}$ Number of observations in final fully adjusted model

b Of complete responses

smoking. Finally, respondents had 15 times the odds of reporting exposure to tobacco advertising if they were at a store (aOR 14.82, 95\% CI 3.61, 60.88) compared to when they were at home. In adjusted analyses, no other locations were significantly different than home.

\section{Discussion}

When controlling for other factors, African American and Hispanic participants are more likely to report exposure to tobacco advertising. Respondents were also significantly more likely to report exposure to advertisements in stores. A large body of research has found increased tobacco advertising in stores in minority neighborhoods $[8-10,21,22]$ suggesting that greater exposure among minority respondents may be due to more advertising in the environment. Point-of-sale tobacco marketing exposure may be particularly problematic, as exposure in stores, but not through other media, has been associated with increased future smoking risk [23]. Additionally, store type where respondents shop may play a role in these results, with convenience stores compared with other store types like grocery stores, more likely to have tobacco marketing [24]. We did not ask respondents who were at stores at EMA response to report the type of store visited, though this or automated geolocation monitoring through the smartphone could be added to future studies. However, though significant, these results should be interpreted cautiously and replicated in larger studies due to low sample sizes and wide confidence intervals. 
Respondents were also more likely to report advertising exposure when in a group or in view of others using tobacco products. This finding is concerning as a prior EMA study of young adults found that being with a friend when exposed to pro-tobacco marketing was associated with greater intention to smoke in the future compared with exposure when alone, which was unrelated to intention [25]. Additionally, experiencing tobacco advertising in places where tobacco use is present may both serve as cues for use [26,27]. Multiple consistent 'social exposures' (such as smoking and tobacco marketing) may interact to make tobacco use more normative which can in turn influence smoking risk [28].

\section{Strengths and limitations}

This study has several limitations. The small sample size for the pilot study precludes broad generalizations and the study sample was predominately female; however, it did include a relatively diverse sample and is in line with the sample size in other pilot EMA studies of tobaccorelated behaviors $[25,29,30]$. This study also focused on responses to random prompts, rather than also incorporating user-initiated responses as in other studies of marketing exposure $[19,25]$. This approach may, therefore, have missed some advertising exposures, however, it reduces potential bias introduced by study-induced heightened participant awareness of tobacco marketing, which has been associated with increased susceptibility [31] and future use [16].

Strengths of the study are the wide applicability of the text messaging approach due to $85 \%$ smartphone ownership among young adults and $100 \%$ of text messaging use among smartphone owners in that age group [32]. Additionally, the response to the random prompts was relatively high, comparable with other studies of youth or young adults and generated nearly 60 observations on average per respondent [33, 34]. Momentary reporting of tobacco marketing exposure reduces recall bias and was more strongly correlated with future susceptibility than recall measures [19]. Additionally, the self-reported measure was broad-based in assessing marketing exposure through multiple channels and not just at the point-of-sale.

This pilot study finds that this EMA approach is feasible to engage young adult non-smokers in reporting on their momentary exposure to tobacco marketing. We intend to develop a larger study which examines differences in exposure between vulnerable population young adults and non-vulnerable young adults. This future study will assess whether differential neighborhood and individual exposure to tobacco marketing is associated with greater susceptibility to future use of tobacco, tobacco use disparities in vulnerable groups, and greater likelihood of tobacco use initiation over time.

\section{Conclusions}

This pilot study shows that both susceptible and non-susceptible young adult nonsmokers are routinely exposed to tobacco marketing in their daily life, particularly at the point-of-sale. EMA protocols can be effective in assessing the potential impact of point-of-sale tobacco marketing on young adults. Regulations on point-of-sale marketing [35] may reduce exposure for young adults.

\section{Additional file}

Additional file 1: Appendix A.Baseline study instrument.

Abbreviations

aOR: adjusted odds ratio; Cl: confidence interval; EMA: ecological momentary assessment.

\section{Authors' contributions}

SWR designed the study and was in charge of all aspects of data collection and analysis and served as lead author of the manuscript. HE conducted statistical data analyses. AAR and RN provided input into study design and analytic plan. All authors provided substantive contributions to the development and editing of the manuscript and approved the final manuscript. All authors read and approved the final manuscript.

\section{Author details \\ ${ }^{1}$ Schroeder Institute at Truth Initiative, Washington, DC, USA. ${ }^{2}$ College of Global Public Health, New York University, New York, NY, USA.}

\section{Acknowledgements}

The authors would like to thank Ashley Mayo for assisting with participant recruitment and monitoring, Morgane Bennett for assistance with the EMA system, and Thomas Kirchner for consultation with the pilot study protocol.

\section{Competing interests}

The authors declare that they have no competing interests.

Availability of data materials

De-identified data are available upon request from the study authors.

\section{Consent for publication}

Participants provided written informed consent to publish results.

\section{Ethics, approval, and consent to participate}

The study was approved by the Chesapeake Institutional Review Board (Study Number \#Pro00012367). All respondents provided written and signed consent to participate consistent with this approved study protocol.

\section{Funding}

This study was funded by Truth Initiative and the funder did not have a role in the design of the study and collection, analysis, and interpretation of data or in writing the manuscript.

\section{Publisher's Note}

Springer Nature remains neutral with regard to jurisdictional claims in published maps and institutional affiliations.

Received: 4 January 2017 Accepted: 23 August 2017

Published online: 31 August 2017 


\section{References}

1. Fulmer EB, Neilands TB, Dube SR, Kuiper NM, Arrazola RA, Glantz SA. Protobacco media exposure and youth susceptibility to smoking cigarettes, cigarette experimentation, and current tobacco use among US youth. PLOS ONE. 2015;10(8):e0134734.

2. Gilpin EA, White MM, Messer K, Pierce JP. Receptivity to tobacco advertising and promotions among young adolescents as a predictor of established smoking in young adulthood. Am J Public Health. 2007;97(8):1489-95

3. Shadel WG, Martino SC, Setodji C, Scharf D. Momentary effects of exposure to prosmoking media on college students' future smoking risk. Health Psychol. 2012;31(4):460-6.

4. Ling PM, Neilands TB, Glantz SA. Young adult smoking behavior: a national survey. Am J Prev Med. 2009;36(5):389-94.

5. Brown A, Moodie C. The influence of tobacco marketing on adolescent smoking intentions via normative beliefs. Health Educ Res. 2009;24(4):721-33.

6. DiFranza JR, Wellman RJ, Sargent JD, et al. Tobacco promotion and the initiation of tobacco use: assessing the evidence for causality. Pediatrics. 2006;117(6):e1237-48

7. Wellman RJ, Sugarman DB, DiFranza JR, Winickoff JP. The extent to which tobacco marketing and tobacco use in films contribute to children's use of tobacco: a meta-analysis. Arch Pediatr Adolesc Med. 2006;160(12):1285-96

8. Moreland-Russell S, Harris J, Snider D, Walsh H, Cyr J, Barnoya J. Disparities and menthol marketing: additional evidence in support of point of sale policies. Int J Environ Res Public Health. 2013;10(10):4571-83.

9. Widome R, Brock B, Noble P, Forster JL. The relationship of neighborhood demographic characteristics to point-of-sale tobacco advertising and marketing. Ethn Health. 2013;18(2):136-51

10. Henriksen L, Schleicher NC, Dauphinee AL, Fortmann SP. Targeted advertising, promotion, and price for menthol cigarettes in California high school neighborhoods. Nicotine Tob Res. 2012;14(1):116-21.

11. Dauphinee AL, Doxey JR, Schleicher NC, Fortmann SP, Henriksen L. Racial differences in cigarette brand recognition and impact on youth smoking. BMC Public Health. 2013;13:170

12. Soneji S, Ambrose BK, Lee W, Sargent J, Tanski S. Direct-to-consumer tobacco marketing and its association with tobacco use among adolescents and young adults. J Adolesc Health. 2014;55(2):209-15.

13. Shiffman S. Ecological momentary assessment (EMA) in studies of substance use. Psychol Assess. 2009;21(4):486-97.

14. Shiffman S, Stone AA, Hufford MR. Ecological momentary assessment. Annu Rev Clin Psychol. 2008;4:1-32.

15. Siahpush M, Shaikh RA, Hyland A, et al. Point-of-sale cigarette marketing, urge to buy cigarettes, and impulse purchases of cigarettes: results from a population-based survey. Nicotine Tob Res. 2016;18(5):1357-62.

16. Henriksen L, Schleicher NC, Feighery EC, Fortmann SP. A longitudinal study of exposure to retail cigarette advertising and smoking initiation. Pediatrics. 2010;126(2):232-8.

17. Soong A, Chen JC, Borzekowski DL. Using ecological momentary assess ment to study tobacco behavior in urban India: there's an app for that. JMIR Res Protoc. 2015;4(2):e76

18. Kirchner TR, Cantrell J, Anesetti-Rothermel A, Ganz O, Vallone DM, Abrams DB. Geospatial exposure to point-of-sale tobacco: real-time craving and smoking-cessation outcomes. Am J Prev Med. 2013:45(4):379-85.
19. Martino SC, Scharf DM, Setodji CM, Shadel WG. Measuring exposure to protobacco marketing and media: a field study using ecological momentary assessment. Nicotine Tob Res. 2012;14(4):398-406.

20. Strong DR, Hartman SJ, Nodora J, et al. Predictive validity of the expanded susceptibility to smoke index. Nicotine Tob Res. 2015;17(7):862-9.

21. Roberts ME, Berman ML, Slater MD, Hinton A, Ferketich AK. Point-of-sale tobacco marketing in rural and urban Ohio: could the new landscape of tobacco products widen inequalities? Prev Med. 2015;81:232-5.

22. Lee JG, Henriksen L, Rose SW, Moreland-Russell S, Ribisl KM. A systematic review of neighborhood disparities in point-of-sale tobacco marketing. Am J Public Health. 2015;105(9):e8-18.

23. Shadel WG, Martino SC, Setodji C, Scharf D. Exposure to pro-smoking media in college students: does type of media channel differentially contribute to smoking risk? Ann Behav Med. 2013:45(3):387-92.

24. Center for Public Health Systems Science. Point-of-sale report to the nation: realizing the power of states and communities to change the tobacco retail and policy landscape. St. Louis, MO: center for public health systems science at the Brown School at Washington university in St. Louis and the national cancer institute, state and community tobacco control research initiative; 2016.

25. Scharf DM, Martino SC, Setodji CM, Staplefoote BL, Shadel WG. Middle and high school students' exposure to alcohol- and smoking-related media: a pilot study using ecological momentary assessment. Psychol Addict Behav. 2013;27(4):1201-6.

26. Voorhees CC, Ye C, Carter-Pokras O, et al. Peers, tobacco advertising, and secondhand smoke exposure influences smoking initiation in diverse adolescents. Am J Health Promot. 2011;25(3):e1-11.

27. Gilpin EA, White VM, Pierce JP. How effective are tobacco industry bar and club marketing efforts in reaching young adults? Tob Control. 2005;14(3):186-92.

28. Mead EL, Rimal RN, Ferrence R, Cohen JE. Understanding the sources of normative influence on behavior: the example of tobacco. Soc Sci Med. 2014:115:139-43.

29. Bujarski S, Roche DJ, Sheets ES, Krull JL, Guzman I, Ray LA. Modeling naturalistic craving, withdrawal, and affect during early nicotine abstinence: a pilot ecological momentary assessment study. Exp Clin Psychopharmacol. 2015;23(2):81-9.

30. Gwaltney CJ, Bartolomei R, Colby SM, Kahler CW. Ecological momentary assessment of adolescent smoking cessation: a feasibility study. Nicotine Tob Res. 2008:10(7):1185-90.

31. Feighery E, Borzekowski DL, Schooler C, Flora J. Seeing, wanting, owning: the relationship between receptivity to tobacco marketing and smoking susceptibility in young people. Tob Control. 1998;7(2):123-8.

32. Smith A, Page D. US smartphone use in 2015. 2015.

33. Buckner JD, Crosby RD, Silgado J, Wonderlich SA, Schmidt NB. Immediate antecedents of marijuana use: an analysis from ecological momentary assessment. J Behav Ther Exp Psychiatry. 2012;43(1):647-55.

34. Hedeker D, Mermelstein RJ, Berbaum ML, Campbell RT. Modeling mood variation associated with smoking: an application of a heterogeneous mixed-effects model for analysis of ecological momentary assessment (EMA) data. Addiction. 2009:104(2):297-307.

35. Lange T, Hoefges M, Ribisl KM. Regulating tobacco product advertising and promotions in the retail environment: a roadmap for states and localities. J Law Med Ethics. 2015;43(4):878-96. 